\title{
Scattering by an array of perforated cylinders with a porous core
}

\author{
Bart van der $\mathrm{Aa}^{\mathrm{a})}$ and Jens Forssén \\ Division of Applied Acoustics, Chalmers University of Technology, SE-41296 Gothenburg, Sweden
}

(Received 22 April 2014; revised 5 August 2014; accepted 16 September 2014)

\begin{abstract}
In this work multiple scattering by an array of perforated cylindrical shells with a porous core has been investigated. A semi-analytical model to predict scattering from such cylindrical units is presented in the context of the multiple scattering theory (MST), and validated against laboratory experiments. The suggested semi-analytical multiple scattering model uses an impedance expression to include the perforated shell in the scattering coefficients, which is a compact way to describe a composite scatterer in MST. Calculation results of a small array are shown to be in excellent agreement with measured data. Predictions and data show that perforated cylinders with empty cavities exhibit a strong and narrow insertion loss peak at resonance, though simultaneously suffer from amplification below resonance. By adding porous material in the core of the scatterer adverse effects below the resonance peak were suppressed. In addition, it was found that the reduction peak broadens, though at a cost of a reduced peak amplitude. Finally, it has been shown that adding porous material in a perforated shell will introduce partial absorption of the incoming field, which can be optimized by adjusting the perforation ratio of the shell.
\end{abstract}

(C) 2014 Acoustical Society of America. [http://dx.doi.org/10.1121/1.4896566]

PACS number(s): 43.20.Fn, 43.20.Ks, 43.50.Gf [OU]

Pages: $2370-2380$

\section{INTRODUCTION}

Over the last few decades, studies dedicated to periodic arrangements of scattering objects have acquired considerable attention among scientists, and became an important research topic in academic fields such as optics, ocean engineering, and acoustics. The common goal in these various fields is to understand and, ultimately, fully control wavepropagation through an array of periodically spaced scattering objects. In acoustics, one often classifies a periodic arrangement of scatterers as a sonic crystal (SC) or phononic crystal, the latter being derived from its electro-magnetic counterpart, a photonic crystal.

Studies on wave interaction with SC structures can, roughly, be divided into two main classes: (i) where wavelengths are much larger than the periodic length of the structure, and (ii) where wavelengths are in the order of the periodic length of the structure, or shorter. The first class of studies is mostly concerned with focusing and redirection of waves, ${ }^{1,2}$ whereas the second class mostly utilizes stop-band phenomena. $^{3-5}$ Infinite periodic SC structures have frequency intervals where waves cannot propagate, which are the stop-band or band-gap regions. Strictly speaking, band-gaps only exist in infinite structures, though frequency intervals of very low acoustic transmission exist in finite structures. ${ }^{6}$ The lowest band-gap center frequency, associated with Bragg diffraction, is typically found when the wavelength of the impinging wave approaches twice the largest periodic length of the structure.

The performance of sonic crystal structures and its operating frequency interval can be improved, or adjusted, by alternating the scatterer arrangement, the filling fraction, or by introducing scattering units with physical boundary

\footnotetext{
${ }^{\text {a) }}$ Author to whom correspondence should be addressed. Electronic mail: bart.vanderaa@chalmers.se
}

conditions other than acoustically rigid. In the recent past, several studies on two-dimensional SC structures with locally resonant cylindrical units have been conducted. $^{7-11}$ A straightforward example of locally resonant elements is, e.g., split-ring resonators. ${ }^{8,9}$ These slotted cylindrical objects have a cross-section that is approximately invariant along the main axis of the cylinder, and are essentially two-dimensional Helmholtz resonators. ${ }^{9}$ Other types of locally resonant elements such as, for instance, Matryoshka type cylindrical scatterers, ${ }^{7}$ elastic shells, ${ }^{12}$ concentric split-ring resonators with inner elastic-shells, ${ }^{9}$ perforated bamboo rods, ${ }^{10}$ microperforated cylinders, ${ }^{13}$ and elastic U-shaped scatterers, ${ }^{11}$ have also been studied. All locally resonant scatterers mentioned above share the feature to introduce at least one complete band-gap due to an acoustic and/or mechanical resonance of the scatterer, which is typically found below the lowest bandgap associated with Bragg diffraction from the lattice. An alternative approach to improve the performance of a SC structure, by adjusting the scattering units, is to add a porous cover on the outside of an acoustically rigid cylindrical core. ${ }^{14}$ Adding porous material on the outside of the scatterer has been shown to make the insertion loss spectra as a function of frequency more uniform. ${ }^{14}$ Combining the concept of split-ring resonators covered with a porous layer on the outside of the scatterer has also been studied experimentally and numerically. ${ }^{15}$ It has been shown that such composite scatterers may simultaneously benefit from several attenuating phenomena, which allows engineers to tailor the insertion loss of, e.g., a noise barrier. ${ }^{15}$ However, a possible drawback of adding a porous material on the outside of a scatterer is, e.g., its durability with regards to mechanical impacts.

In this work multiple-scattering by an array of perforated cylindrical shells with a porous core will be investigated. The shells have circular perforations, which are periodically distributed in the longitudinal and circumferential direction. Porous material is added in the cavity of the 
hollow cylinder, and is in close contact with the shell surface. Since porous material is located in the interior of the shell it is protected from mechanical impacts. A similar strategy, where thin metal shells are filled with rubber crumb, has been studied by the authors of Ref. 16. However, they simplified the $T$-matrix formulation of the composite scatterer by assuming that the perforated shells surrounding the rubber crumb are acoustically transparent. In this work, we will formulate the full problem of a composite scatterer made of perforated shells with or without a porous core. Consequently, the perforation-ratio of the shell and the shellthickness can be taken into account, dropping the assumption of an acoustically transparent shell. Ultimately, these parameters could be optimized to maximize the insertion loss of, e.g., noise barriers. A semi-analytical theoretical model, applicable in the kernel of multiple scattering theory (MST), is presented and validated against laboratory experiments. We implicitly include the perforated cylindrical shell in the scattering coefficients of MST, by coupling the inner and outer domain of the scatterer through an impedance formulation similar to Ref. 13. Calculation results have been verified with experimental data for an array of $2 \times 3$ scatterers. In order to give an outlook of expected results for larger arrays, band-structure calculations for infinite structures as well as insertion loss spectra for finite arrays of $10 \times 3$ scatterers are presented. In addition, we present absorption, reflectance and transmittance spectra for a finite array of $150 \times 3$ cylindrical units, where the perforation ratio of the shell has been varied. In the next section, an outline of the theoretical model is given first.

\section{THE MODEL}

Let us first consider a single infinitely long perforated cylinder with outer radius $r_{o}$, inner radius $r_{i}$, shell thickness $h$, and a periodic distribution of circular perforations with hole radius $r_{h}$, see Fig. 1 . The medium exterior to the cylinder starts when $r>r_{o}$, with $r$ a two-dimensional vector measured from the origin $o$ of the scatterer. Position vectors are expressed in polar coordinates $(r, \theta)$ or Cartesian coordinates $(x, z)$. The interior domain starts when $r<r_{i}$, and acoustic propagation therein is described by a complex density $\rho_{i}$, and complex sound speed $c_{i}$. Acoustic propagation in the exterior domain, on the other hand, is characterized by the density of air $\rho_{0}$, and sound speed of air $c_{0}$. Hence, in the special case when $\rho_{i}=\rho_{0}$ and $c_{i}=c_{0}$, the configuration reduces to a perforated shell facing air on both sides. It is assumed that waves propagate perpendicular to the
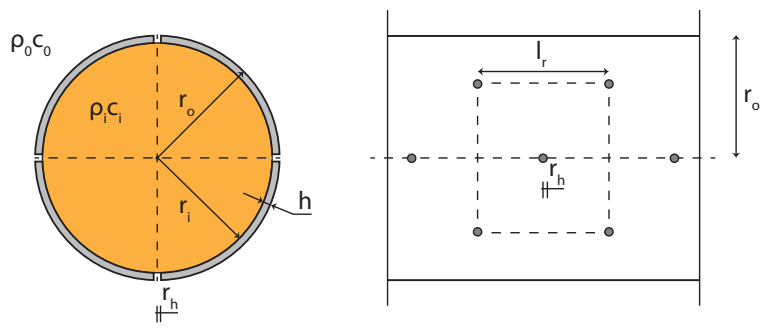

FIG. 1. (Color online) Cross-section of a perforated cylinder (left panel) and a side- view illustration from a section of the cylinder (right panel). longitudinal axis of the infinite cylindrical object, with origin $o$. If not explicitly stated otherwise, a source with cylindrical symmetry, and $e^{-i \omega t}$ time-dependence, has been assumed throughout the paper.

In the low-frequency region a cylindrical shell with evenly distributed perforations or slots can be approximated by a homogeneous fluid layer of thickness $h$, which is characterized by its effective density $\rho_{l}$, and effective sound speed $c_{l}$. By applying appropriate boundary conditions on both faces of the cylinder and coupling the infinite series of Bessel and Hankel functions describing acoustic propagation in the interior, fluid shell, and exterior domains, one can solve for scattering coefficients of the fluid shell. ${ }^{9}$ Similar approximations have also been presented for slotted (twodimensional) cylinders, and perforated (three-dimensional) circular scatterers by other groups. ${ }^{17}$ The latter study presented limiting criteria for the low-frequency assumption of periodically distributed Helmholtz resonators to be valid, which, in turn, was based on work presented in Ref. 18. It has been postulated in Ref. 9 that the approximate solution of a slotted cylinder finds the first resonance position with a maximum error of $5 \%$, provided that

$$
\phi<\frac{1}{4} \quad \text { and } \quad \lambda=\frac{c}{f}>\phi,
$$

where $\phi$ is the filling fraction of the openings. For a slotted cylinder (that is invariant in the longitudinal direction) $\phi=\mathcal{N} d_{n} /\left(2 \pi r_{o}\right)$, with $\mathcal{N}$ a number of equally spaced slots. However, for a perforated cylinder, $\phi$ is based on the threedimensional cylinder geometry, and can be found by evaluating a cylinder face unit-cell, which is indicated as a dashed rectangle in the right-panel of Fig. 1. In essence the ratio of the opening surface to the unit-cell surface needs to be calculated. For a perforated cylinder having four holes in a circumferential plane and using a triangular hole distribution in the longitudinal direction,

$$
\phi<\frac{4 r_{n}^{2}}{l_{r} r_{o}}
$$

where $l_{r}$ is the perforation separation length in the longitudinal direction, see Fig. 1. Although we used the perforation arrangement as explained, the model we will present subsequently can be used for other arrangements as long the distribution is periodic in both circumferential and longitudinal direction. In addition, the criteria shown in Eq. (3) must be met.

Instead of formulating acoustic propagation in the inner, outer and shell domains explicitly, it has been shown that one can substitute the infinite series of Bessel and Hankel functions of the shell domain with an impedance formulation. ${ }^{13}$ We will follow the approach initiated in that paper and present a suitable impedance formulation of the perforated shell and corresponding scattering coefficients for the problem at hand.

\section{A. Acoustic impedance of a perforated plate}

A plate with circular perforations can be seen as a collection of tubes surround by a rigid frame. Due to 
air-movement inside these tubes a pressure drop $\Delta p$ takes places across the length of the tubes. Assuming that perforations are separated by a distance much larger than the opening diameter, though much smaller than the wavelength of interest, the specific impedance without end correction terms is given by, e.g., ${ }^{19-21}$

$$
Z_{h}=\frac{\Delta p}{v}=\frac{i \omega \rho_{0} h}{\phi}\left[1-\frac{2}{s \sqrt{-i}} \frac{J_{1}(s \sqrt{-i})}{J_{0}(s \sqrt{-i})}\right]^{-1},
$$

where $\omega$ is the angular frequency, $v$ is the average velocity over the tube cross section, $i^{2}=-1, J_{n}(\cdots)$ is the Bessel function of the first kind and order $n$, and $s$ is the perforation constant given by $s=r \sqrt{\rho_{0} \omega / \eta_{0}}$, in which $\eta_{0}$ is the dynamic viscosity of air. In addition to internal resistive and reactive effects, external effects take place due to air movement outside the holes. To account for external effects a combination of resistive and reactive end-correction terms is often introduced. We are interested in two sets of endcorrection terms: (i) where the acoustic medium inside and outside of the cylindrical shell is air, and (ii) where the acoustic medium inside the cylindrical shell is a porous material and outside is air. The resistive end-correction of the first case can be introduced in terms of power dissipation due to an oscillatory flow over an infinite plane surface. ${ }^{22}$ For such a configuration the theoretical expression of the surface resistance is given by $R_{s}=\frac{1}{2} \sqrt{2 \eta_{0} \rho_{0} \omega}$, see, e.g., Ref. 22. The added surface resistance of a perforated plate would naturally be $2 R_{s}$. Though based on measurements $4 R_{s}$ is often chosen, see, e.g., Ref. 22 . In this work $2 R_{s}$ will be used as the resistive end-correction term for the first case. The total reactive end-correction is composed of an outer term $\delta_{0}=8 r / 3 \pi$, and inner term $\delta_{i}=0.48 \sqrt{\pi r^{2}}(1$ $-1.25 \sqrt{\phi}) .{ }^{22,23}$ Grouping the end correction terms of the first case, i.e., those for an empty cavity, we may write

$$
Z_{e}=\frac{\sqrt{2 \eta_{0} \rho_{0} \omega}}{\phi}+\frac{i \omega \rho_{0}\left(\delta_{0}+\delta_{i}\right)}{\phi} .
$$

Hence, the total specific impedance of a perforated shell in contact with air on both sides is given by

$$
Z_{t}=Z_{h}+Z_{e}
$$

Next we seek for a set of end correction terms to adjust the impedance of a perforated shell where one side is in contact with a porous material. Since the outer end-correction term is given above, we look here for the inner end-correction term only. It has been shown by several authors that when a porous layer is in contact with the perforated facing the inner-end correction becomes complex. ${ }^{24,25}$ This means that the porous layer adds a resistive component to the total specific impedance, other than the surface resistance as discussed before. By a simple substitution it is possible to rewrite the impedance expression of the reactive inner end-correction in terms of $k_{0}$ and $\rho_{0} c_{0}$. After that, one can substitute the properties of air for the acoustical properties of the porous medium and arrive at

$$
Z_{e^{\prime}}=\frac{\sqrt{\eta_{0} \rho_{0} \omega}}{\phi}+\frac{i \omega \rho_{0} \delta_{o}}{\phi}+\frac{i k_{i} \rho_{i} \delta_{i}}{\phi} .
$$

In brief, we recognize the surface resistance as the first term of the right-hand side, the reactive outer end-correction as the second, and the inner end-correction as the third. Notice that the latter is now written as a function of the characteristic impedance and complex wavenumber of the porous material. These may be obtained by simple impedance models such as the empirically obtained Delany and Bazley model, ${ }^{26}$ the Miki model, ${ }^{27}$ or more sophisticated models such as, e.g., the relaxation model for porous media by Wilson. ${ }^{28}$ Now, the total specific impedance of a perforated shell in contact with air on one side and a porous material on the other side is given by

$$
Z_{t^{\prime}}=Z_{h}+Z_{e^{\prime}}
$$

It should be noted that viscous losses around the openings are generally much smaller than the resistive component of the impedance $Z_{h}$ and hence dropping these terms leads in many cases to marginal differences. Note also that no hole interaction effects are included in the impedance formulations as the perforation ratio is low, here $\phi \approx 0.016$.

\section{B. Auxiliary problem: scattering by a single perforated cylinder}

The theoretical model to be presented subsequently is developed in the framework of MST. ${ }^{4,29,30}$ In this method, scattering by a cluster of non-overlapping infinitely long cylindrical (or spherical in three-dimensions) objects can be formulated efficiently in a semi-analytical approach. The infinite summation series of Bessel and Hankel functions to express the incoming and scattered fields usually converge fast, which has lead to a broad interest in MST up and until to-date. Though conceptually similar, various notational conventions could be used to formulate a multiple scattering problem. We will follow the convention as used in, e.g., Ref. 9. For convenience we first introduce an auxiliary problem to derive scattering coefficients for a single perforated cylinder as shown in Fig. 1. The scattered field at a receiver for which $\hat{r}>r_{o}$ can be expressed in a series of Hankel and trigonometric functions, and may be written

$$
p_{o}^{s}(r, \theta)=\sum_{n=-\infty}^{\infty} A_{n} H_{n}\left(k_{0} \hat{r}\right) \exp (i n \hat{\theta}), \quad \hat{r}>r_{o}
$$

where $H_{n}(\cdots)$ is the Hankel function of first kind and order $n, k_{0}=\omega / c_{0}$ the wavenumber in air, and $A_{n}$ are unknown coefficients. In addition, notice that the receiver coordinate system $(\hat{r}, \hat{\theta})$ is here seen from the origin of the scatterer. The total acoustic field may now be written as

$$
p_{o}(r, \theta)=H_{0}\left(k_{0} r\right)+p_{o}^{s}(r, \theta)
$$

where $H_{0}\left(k_{0} r\right)$ is the incoming pressure from the source. Using Graf's addition theorem for Bessel functions $H_{0}\left(k_{0} r\right)$ 
can be expanded about the origin of the scatterer, and Eq. (9) may be rewritten as

$$
\begin{aligned}
p_{o}(\hat{r}, \hat{\theta})= & \sum_{n=-\infty}^{\infty}\left[H_{n}\left(k_{0} R\right) J_{n}\left(k_{0} \hat{r}\right) \exp (-i n(\pi+\xi))\right. \\
& \left.+A_{n} H_{n}\left(k_{0} \hat{r}\right)\right] \exp (i n \hat{\theta}), \quad \hat{r}>r_{o}
\end{aligned}
$$

where $\mathbf{R}=R(\cos \xi, \sin \xi)$ is a radius vector to the cylinder origin. The acoustic field in the interior region of the cylindrical shell, i.e., when $\hat{r}>r_{o}$, can be expressed as

$$
p_{i}(r, \theta)=\sum_{n=-\infty}^{\infty} B_{n} J_{n}\left(k_{0} \hat{r}\right) \exp (i n \hat{\theta}), \hat{r}>r_{i}
$$

where $k_{i}=\omega / c_{i}$, and $B_{n}$ are unknown coefficients. We will now derive scattering coefficients of a perforated shell in a similar fashion as has been presented in Ref. 13. The basic idea is to couple the inner and outer domain of the cylinder through a cylinder shell impedance $Z_{t}$, which has been defined previously. Assuming that the normal velocity with respect to the shell surface is constant across $h$, and using the impedance relation between pressure and velocity we may write

$$
v_{o}\left(r_{o}\right)=v_{i}\left(r_{i}\right)
$$

and

$$
v_{o}\left(r_{o}\right)=\frac{p_{o}\left(r_{o}\right)-p_{i}\left(r_{i}\right)}{Z_{t}}
$$

where $v_{o}$ and $v_{i}$ are the normal velocities at the outer and inner shell faces, respectively. The normal velocities and the spatial pressure gradient can be coupled through conservation of momentum,

$$
\nabla p_{\alpha}=-i \omega \rho_{\alpha} v_{\alpha}
$$

where $\nabla p_{\alpha}$ is the spatial pressure gradient, $\rho_{\alpha}$ the medium density, $v_{\alpha}$ the normal velocity, and $\alpha=i$, o. Using orthogonality of the expanded terms, i.e., multiplication of $p_{\alpha}$ with $\exp (-\operatorname{im} \theta)$, in which $m$ is an integer, and integration over $\theta$ from $0 \rightarrow 2 \pi$ gives

$$
\begin{aligned}
\left\langle p_{\alpha}, \exp (-i m \theta)\right\rangle & =\int_{0}^{2 \pi} p_{\alpha} \exp (-i m \theta) d \theta \\
& = \begin{cases}2 \pi \sum_{n=-\infty}^{\infty}\{\cdots\}, & m=n \\
0, & m \neq n .\end{cases}
\end{aligned}
$$

Substituting Eq. (12) into Eq. (14) and using the definition of Eq. (15) we can write

$$
\begin{aligned}
& \left.\frac{\partial\left\langle p_{\alpha}, \alpha \exp (-i m \theta)\right\rangle}{\partial r}\right|_{r=r_{\alpha}} \\
& =-i \omega \rho_{\alpha}\left[\frac{\left\langle p_{o}\left(r_{o}\right), \exp (-i m \theta)\right\rangle-\left\langle p_{i}\left(r_{i}\right), \exp (-i m \theta)\right\rangle}{Z_{t}}\right] .
\end{aligned}
$$

Using the formulas as given above we can solve for the unknown coefficients $A_{n}$, which are found to be

$$
A_{n}=-Z_{n} H_{n}\left(k_{0} R\right) \exp (-i n(\pi+\xi)),
$$

in which

$$
Z_{n}=\frac{X_{n} J_{n}^{\prime}\left(k_{0} r_{o}\right)-J_{n}\left(k_{0} r_{o}\right)}{X_{n} H_{n}^{\prime}\left(k_{0} r_{o}\right)-H_{n}\left(k_{0} r_{o}\right)}
$$

with

$$
X_{n}=\frac{\rho_{i} k_{0} J_{n}\left(k_{i} r_{i}\right)}{\rho_{0} k_{i} J_{n}^{\prime}\left(k_{i} r_{i}\right)}-\frac{i Z_{t} k_{i}}{\omega \rho_{0}} .
$$

Here, primes are indicating derivatives with respect to $\hat{r}$. It can be noted that the $Z_{n}$ coefficients as presented above capture some of the scatterer types commonly used in the kernel of MST. The scattering coefficients of a fluid cylinder, i.e. a sound permeable cylindrical object, can, e.g., be obtained when the shell thickness $h$ tends to zero and results in $Z_{n}$ as in Eq. (18) though with

$$
X_{n}=\frac{\rho_{i} k_{0} J_{n}\left(k_{i} r\right)}{\rho_{0} k_{i} J_{n}^{\prime}\left(k_{i} r\right)} .
$$

Further, letting the mismatch between the cylinder density $\rho_{i}$ and host medium $\rho_{o}$ to approach infinity, will cause $X_{n}$ to approach infinity and the scattering coefficients of an acoustically rigid cylinder are then obtained,

$$
Z_{n}=\frac{J_{n}^{\prime}\left(k_{0} r\right)}{H_{n}^{\prime}\left(k_{0} r\right)}
$$

For later convenience, we address the fact that the $X_{n}$ terms of the perforated cylindrical shell can be represented alternatively by using the normalized characteristic impedance of the porous layer $Z_{i}$ and than takes the following form:

$$
X_{n}=Z_{i} \frac{J_{n}\left(k_{i} r_{i}\right)}{J_{n}^{\prime}\left(k_{i} r_{i}\right)}-\frac{i Z_{t} k_{i}}{\omega \rho_{0}} .
$$

\section{An array of perforated cylinders}

Let us now consider a cluster of $N$ infinitely long perforated cylinders with outer radius $r_{o}$, inner radius $r_{i}$, and shell thickness $h$. The cylinders are organized in square lattice with lattice constant $a$. Each cylinder has been given a local coordinate system with the origin placed at polar coordinates $\left(\hat{r}_{j}, \hat{\theta}_{j}\right)$, where $j=1, \ldots, N$. Further, we place a receiver at $(r, \theta)$, and a source at $(0,0)$, i.e., the global origin of the domain. If a cylindrical sound wave interacts with a distribution of arbitrarily located cylindrically shaped scatterers the resulting pressure field at any point exterior to the scatterers can be written as

$$
p_{o}(r, \theta)=H_{0}\left(k_{0} r\right)+\sum_{j=1}^{\infty} \sum_{n=-\infty}^{\infty} A_{n}^{j} Z_{n}^{j} H_{n}\left(k_{0} \hat{r}_{j}\right) \exp \left(i n \hat{\theta}_{j}\right),
$$


where the first right-hand side represents the direct field from source-to-receiver, the second term the scattered field from all cylinders to the receiver, and the $Z_{n}^{j}$ coefficients are given by Eq. (18). To solve for the unknown $A_{n}^{j}$ coefficients in Eq. (23), the equation system can be expressed in one set of polar coordinates $\left(\hat{r}_{j}, \hat{\theta}_{j}\right)$. This, again, can be done by using Graf's addition theorem for Bessel functions now applied to Eq. (23). Further, using orthogonality of the terms, i.e., using Eq. (15), and fulfilling the boundary conditions as previously defined leads to an infinite system of equations

$$
\begin{aligned}
A_{m}^{p}+ & \sum_{\substack{j=1 \\
\neq \neq}}^{N} \sum_{n=-\infty}^{\infty} A_{n}^{j} Z_{n}^{j} e^{i(n-m) \xi_{j p}} H_{n-m}^{(1)}\left(k_{0} R_{j p}\right) \\
= & H_{m}\left(k_{0} R_{p}\right) e^{-i m(\pi+\xi \xi p)} \\
& p=1, \ldots, N, \quad m=0, \pm 1, \pm 2, \ldots,
\end{aligned}
$$

where $\mathbf{R}_{\mathbf{j p}}=R_{j p}\left(\cos \xi_{j p}, \sin \xi_{j p}\right)$ is the radius vector from the origin of cylinder $j$ to the origin of cylinder $p$, and $\mathbf{R}_{\mathbf{p}}=R_{p}\left(\cos \xi_{p}, \sin \xi_{p}\right)$ is the radius vector to the origin of the $p$ th cylinder. By truncating the series expansion and rewriting Eq. (24) in the form $\mathbf{M} \times \mathbf{b}=\mathbf{c}$ allows us to solve for the unknown $A_{n}^{j}$ coefficients. The entries of the square matrix $\mathbf{M}=M_{n, m}$ are as follows:

$$
M_{n, m}= \begin{cases}Z_{n}^{j} e^{i(n-m) \xi_{j p}} H_{n-m}\left(k_{0} R_{j p}\right) & \text { if } j \neq p \\ 1 & \text { if } j=p \text { and } n=m \\ 0 & \text { if } j=p \text { and } n \neq m\end{cases}
$$

Here, $\mathbf{M}$ is a square matrix of size $(2 \hat{n}+1) N$ $\times(2 \hat{m}+1) N$, with $\hat{n}=\hat{m}$ and $\hat{n}, \hat{m}$ the maximum value of $|n|,|m|$ after truncation. The vectors $\mathbf{b}$ and $\mathbf{c}$ are $(\hat{m}+1) N$ in size, where $\mathbf{b}$ is filled with $A_{n}^{j}$ coefficients and the source terms in $\mathbf{c}=c_{m}$ are given by

$$
c_{m}=-H_{m}\left(k_{0} R_{p}\right) e^{-i m\left(\pi+\xi_{p}\right)} .
$$

Note that the summation series in Eq. (24) have been truncated such that $|n|,|m|>3 k r_{o}$.

\section{MEASUREMENTS}

\section{A. Geometrical setup and cylinder construction}

In order to verify the theory as introduced in previous sections, an experimental setup consisting of an array of six perforated cylinders with a porous core has been constructed, see Fig. 2.

Measurements are performed in an anechoic chamber measuring $8 \times 8 \times 6 \mathrm{~m}$. The array has been mounted onto a computer controlled turntable and is positioned such that the origin of the array is in line with the speaker opening and microphones. A top-view illustration of the setup is shown in Fig. 3, where zero degrees rotational angle $\varphi$ corresponds to the initial measurement position. Other measurement positions are obtained by clock-wise rotation of the array in increments of $45^{\circ}$. Note that, the source is located at

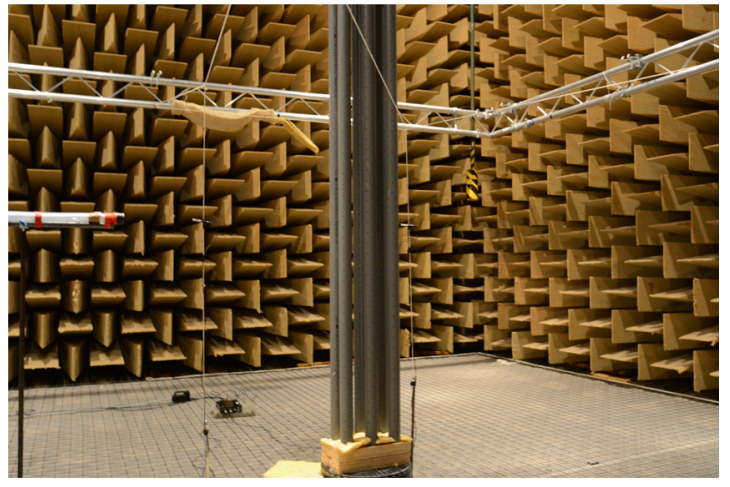

FIG. 2. (Color online) Measurement setup in anechoic chamber.

$\left(x_{s}, z_{s}\right)=(0,0) \mathrm{m}$, microphone 1 at $\left(x_{r, 1}, z_{r, 1}\right)=(0.25,0) \mathrm{m}$, the origin of the array at $\left(x_{a}, z_{a}\right)=(0.85,0) \mathrm{m}$ and microphone position 2 at $\left(x_{r, 2}, z_{r, 2}\right)=(0.25,1.01) \mathrm{m}$.

The cylinders are made from PVC with a shell-thickens $h=3 \mathrm{~mm}$. Each cylinder is $2 \mathrm{~m}$ long and has an outer diameter of $50 \mathrm{~mm}$. The core of the scatterers has been filled with cylindrical pieces of glass-wool. Pieces of material have been cut using a cylindrical cutter and stacked inside the cylinder until completely filled, see Fig. 4. In order to avoid changing material properties due to, e.g., compression, smoothly sliding glass-wool pieces are cut with a diameter being slightly smaller than the actual inner-diameter of the cylinder.

A $90 \mathrm{~mm}$ thick multiplex top-plate and foot-plate with $2 \times 3$ circular holes, matched to the outer diameter of the cylinders, are mounted to both ends and give a firm construction. The cylinders are organized in square lattice with a center-to-center spacing of $10 \mathrm{~cm}$. For measuring we used two B\&K $1 / 4$ in. pair calibrated microphones of type 4135 , which are connected to a pair of G.R.A.S. 26 AC preamplifiers. Up to $5 \mathrm{kHz}$ these microphones have an approximately flat response. The microphones are powered with a G.R.A.S. power module type 12AA, and data acquisition is performed using a National Instruments DAQ of type 9234 and MATLAB based acquisition software. We measured with a frequency resolution of $10 \mathrm{~Hz}$, a block length of $100 \mathrm{~ms}$, and a sampling frequency of $51.2 \mathrm{kHz}$. To irradiate the array, white noise generated by a B\&K 1405 noise generator, amplified by a NAS amplifier, has been used. A source was created using a compression driver connected to a ca. $4 \mathrm{~m}$ long flexible hose with an aluminum ring at the opening. The resulting effective source opening diameter is $25 \mathrm{~mm}$. Both the source opening and the microphones are mounted at around $0.95 \mathrm{~m}$ above the floor-plate. A permanent steel-frame (not visible)

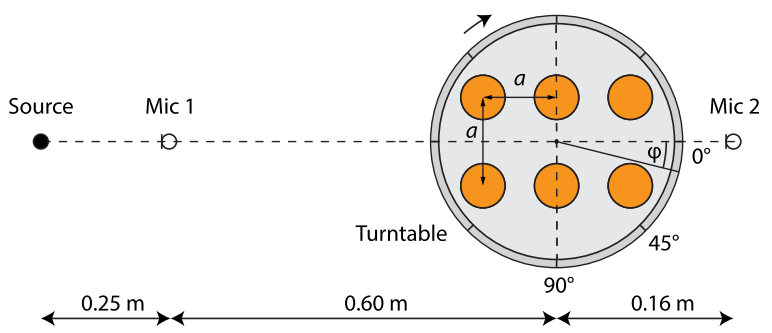

FIG. 3. (Color online) Top-view Illustration of measurement setup. 


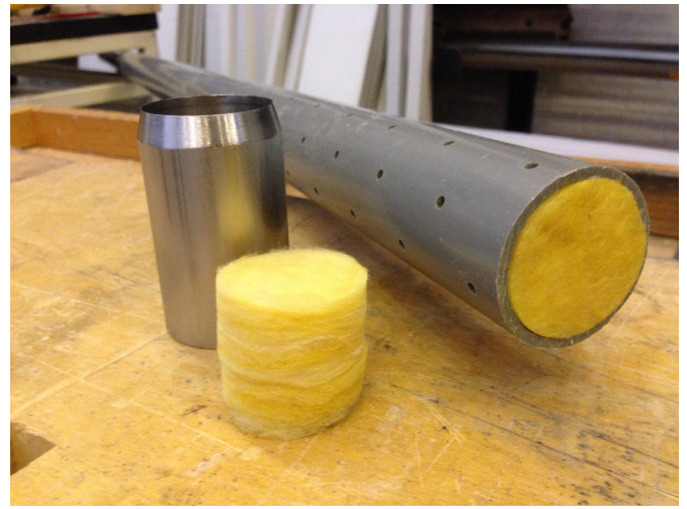

FIG. 4. (Color online) Close-up of perforated cylinder with porous core and glass-wool cutter.

was used to mount the flexible hose, whereas the microphones were attached to a firm web of thin cotton wires.

\section{B. Characterization of porous material}

The cylinder cores are filled with glass-wool, which has unknown acoustical properties. In order to compare calculation results with measured data, the impedance of the material needs to be obtained and serves as input in the theoretical model. Here, we use a single- parameter impedance model based on the flow-resistivity. ${ }^{27}$ Finding the flow resistivity of a test material is often done by fitting the theoretical surface impedance of a porous layer with hardbacking against the measured surface impedance obtained through impedance tube measurements. A similar approach is followed here. Generally, the acoustical surface impedance of a test material is given by

$$
Z_{s}=Z_{0} \frac{1+R}{1-R}
$$

where $R$ is the complex reflection factor at the surface of the test material and $Z_{0}$, the characteristic impedance of air. A standard transfer function method as described in Ref. 31 is used to compute $R$. The impedance tube has an inner diameter of $10 \mathrm{~cm}$, holds test samples of thickness, $d=5 \mathrm{~cm}$, and uses a microphone switching technique (a single microphone is used to measure the transfer function). The surface impedance as given in Eq. (27) is generally applicable for any type of sample mounting condition. However, in case of a porous layer with a hard backing, the surface impedance may be written as follows:

$$
Z_{s}=Z_{i} \operatorname{coth}\left(-i k_{i} d\right),
$$

where $Z_{i}$ and $k_{i}$ are the characteristic impedance and the complex wavenumber of the material, respectively. Now, minimizing the least squares error between Eq. (27) and Eq. (28) by varying $\sigma$, we can find the best fit of $\sigma$ for a given sample. Repeating this experiment five times for five different material samples, a sample averaged $\sigma$ is found and used as input in the theoretical model. One realization of the fitted surface impedance is shown in Fig. 5. The sample averaged $\sigma$ was found to be $15.1 \mathrm{kPa} \mathrm{s} \mathrm{m}^{-2}$ with a standard deviation of $437 \mathrm{~Pa} \mathrm{~s} \mathrm{~m}^{-2}$.

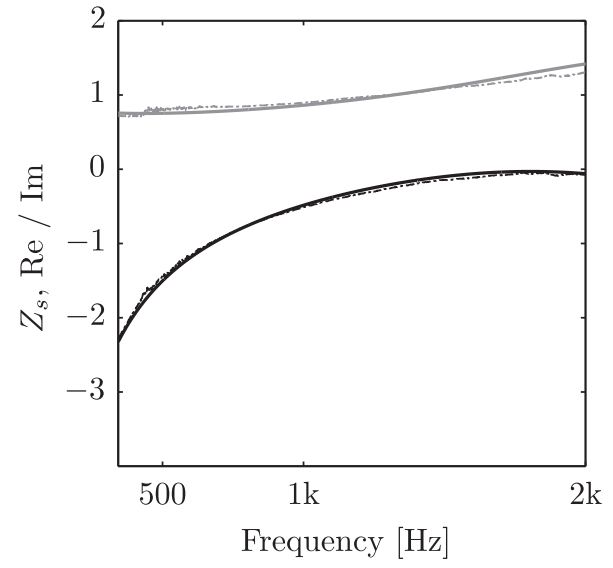

FIG. 5. One realization of measured and fitted surface impedance of a $50 \mathrm{~mm}$ glass-wool sample. The real part of $Z_{s}$ (gray) and the conjugated imaginary part of $Z_{s}$ (black) are plotted for measurements (dashed) and fitted predictions (solid). The best least-squares fit of the surface impedance was found with $\sigma=14.8 \mathrm{kPa} \mathrm{s} \mathrm{m}^{-2}$ in this case.

Notice that the fitted flow resistivity has been obtained with an impedance tube that produces trustworthy results between 340 and $2000 \mathrm{~Hz}$. However, as will be shown in subsequent sections, the main effect of a adding porous material in the core of the scatterer is concentrated well below the $2000 \mathrm{~Hz}$ frequency range. Moreover, the behavior below $340 \mathrm{~Hz}$ is expected to be sufficiently well described by the impedance model. We therefore use $\sigma=15.1 \mathrm{kPa} \mathrm{s} \mathrm{m}^{-2}$ up to $5 \mathrm{kHz}$. Observe also that one could use more sophisticated models to characterize the porous material. However, the agreement of measurements and predictions, which will be demonstrated in the following subsection, justifies the single-parameter model choice in the context of this work. It should, however, be noted that non-physical behavior in the low-frequency region may occur when using the Miki or Delany and Bazley models, as can be read in, e.g., Ref. 32.

\section{Comparison of calculation results with data}

Measurements and calculation results are compared in terms of the insertion loss, which is defined through

$$
\mathrm{IL}=20 \log _{10} \frac{\left|p_{o}^{d}\right|}{\left|p_{o}\right|},
$$

where $p_{o}$ captures the direct field plus scattering from the array and $p_{o}^{d}$ the direct field only. Note that, $p_{o}$ is computed using Eq. (23), and $p_{o}^{d}$ is obtained in closed form through, $H_{o}^{(1)}\left(k_{0} R\right)$. Measurements are performed using a twomicrophone transfer function technique, where the transfer function between the two microphones is obtained with and without array. Substituting the measured transfer function without array for $p_{o}^{d}$ in Eq. (29), and the transfer function with array for $p_{o}$ in Eq. (29), gives the measured IL. Comparisons of measured and computed IL, for perforated cylinders with and without a porous core, and $\varphi=0^{\circ}, 45^{\circ}$, and $90^{\circ}$, are shown in Fig. 6. It can be seen that the agreement for all presented cases is very good as the measurements follow the trend of the predictions closely. Nevertheless, it must be addressed that some spectral 


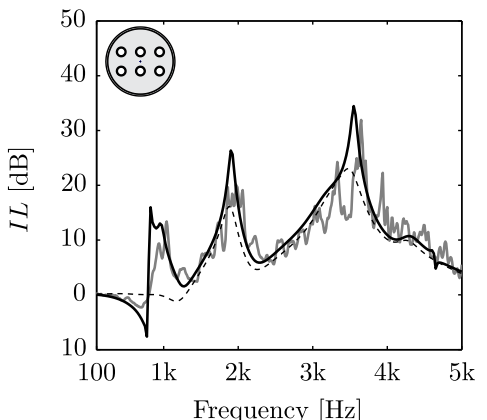

(a)

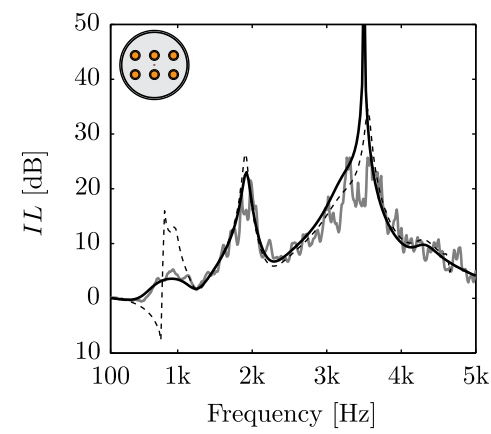

(d)

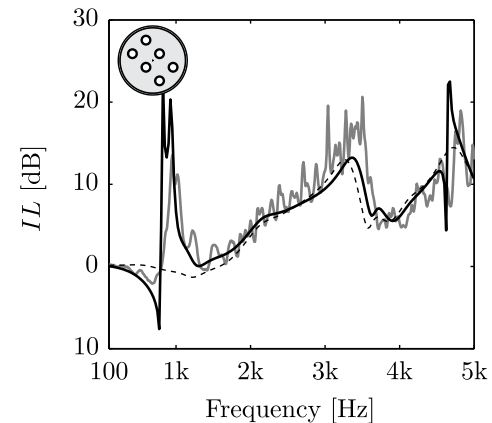

(b)

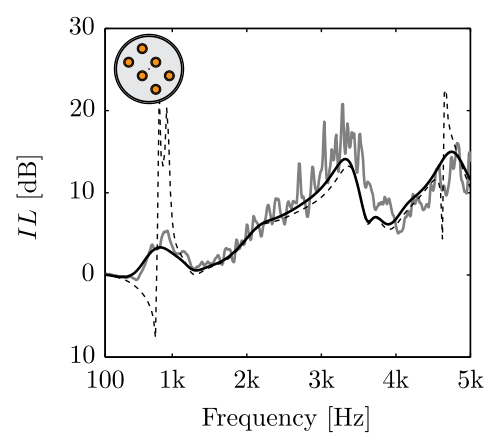

(e)

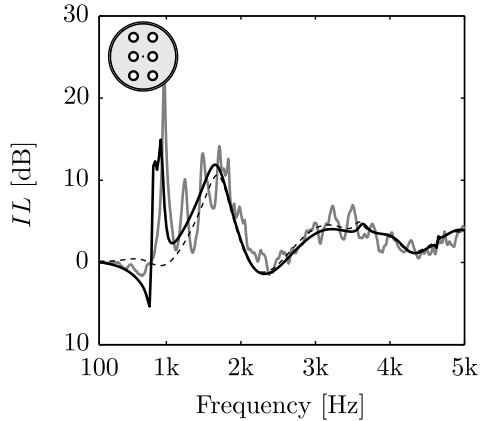

(c)

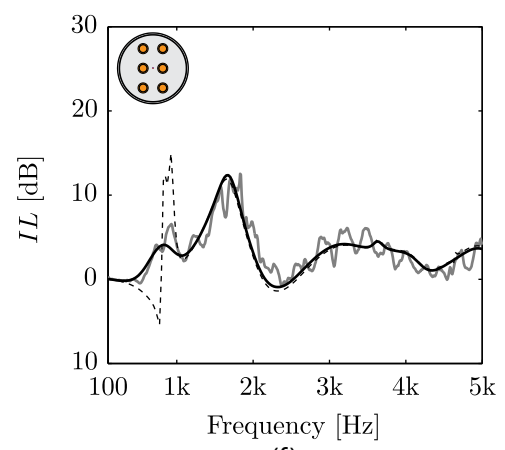

(f)

FIG. 6. (Color online) Insertion loss [dB] comparison between measurements and predictions at $v=0,45$, and $90^{\circ}$. (a)-(c) Measurements of six cylinders with empty cavity (solid-gray), predictions of six perforated cylinders (solid-black), and predictions of six acoustically rigid cylinders (dashed) are plotted. (d)-(f) Measurements of six cylinders with porous core (solid-gray), predictions of six perforated cylinders with porous core (solid-black), and predictions of six perforated cylinders (dashed) are plotted.

oscillations around the mean trend are observed in the measurements, which could not be eliminated. Emphasizing on the array without porous core, i.e., those shown in Figs. 6(a)-6(c), we see that these cylinders introduce a resonance in the transmission spectra at around $1 \mathrm{kHz}$. The peak location of the fundamental resonance is predicted accurately, though the IL dip in the damping and stiffness controlled region is slightly over-predicted by the model. In addition to the fundamental resonance, higher-order resonances in the system exist. The second resonance around $4.7 \mathrm{kHz}$, for instance, is clearly visible in the calculated response at $\varphi=45^{\circ}$. It can be shown that the second resonance is associated to the $(1,1)$ cavity mode, which has one nodal line in the center of the cavity. A more in depth discussion on several mode shapes and associated system properties will be given in Sec. IV B. In Figs. 6(a)-6(c) it is shown that perforated cylinders can improve the IL from and above the resonance frequency of the cylinder. However, below the resonance frequency a significant anti-resonance dip is also observed in the IL spectra. By adding porous material in the core of the scatterer the peak amplitude in the damping controlled region is suppressed, see in Figs. 6(d)-6(f). In addition, it can be seen that the peak width is broadened and now adds a significant reduction below the fundamental resonance frequency. The second resonance, which was observed for cylinders without porous material, is completely suppressed after adding porous material. Thus, for the studied sample, the main positive effect of adding damping material concentrates around the fundamental resonance frequency.

\section{CALCULATION RESULTS}

A multiple scattering formulation for arrays of perforated cylinders with porous cores has been presented and validated in previous sections. The agreement between measured and predicted insertion losses was found to be very good, though spectral oscillations around the mean trend were observed. However, only a small array of $2 \times 3$ cylinders has been studied, which is of limited practical use. To discuss acoustic properties beyond this scope, numerical simulations of an infinite array and a $10 \times 3$ finite array are shown in this section.

The finite array is simply extended in the direction perpendicular to the central source-receiver axis. As such, we can continue to use the source and receiver locations as described previously. Numerical results of the finite arrays are obtained using the model as presented. Band structure calculations of the infinite structures are, on the other hand, obtained using COMSOL MULTIPHYSICS, a commercial finite element method (FEM) solver. The use of COMSOL MULTIPHYSICS to construct band-diagrams has been verified in other studies, see, e.g., Refs. 7 and 11. In addition, we present absorption, reflectance and transmittance spectra for a finite array of $150 \times 3$ cylindrical units, where the perforation ratio of the shell has been varied.

\section{A. Band structure calculations of infinite arrays}

We now consider a two-dimensional array of cylindrical scattering objects, ordered in square lattice. The structure is assumed to be infinite in both $x$ and $y$, with periods $a_{1}$ an $a_{2}$, 
respectively. The origin of the domain $O$ collides with the origin of the central scatterer. Primitive vectors of the lattice are given by $\mathbf{a}_{1}=a_{1} \hat{\mathbf{x}}$ and $\mathbf{a}_{2}=a_{2} \hat{\mathbf{y}}$, in which the unit vectors are defined through $\hat{\mathbf{x}}=(1,0)$ and $\hat{\mathbf{y}}=(0,1)$. It can be shown that the origin $O_{j}$ of scatterer $j$ can be found by the wellknown lattice vector

$$
\mathbf{R}_{j}=n_{1} \mathbf{a}_{1}+n_{2} \mathbf{a}_{2},
$$

where $n_{1}$ and $n_{2}$ are integers. Thus, using the orthogonal primitive vectors plus integers $n_{1}$ and $n_{2}$, all cylinder origins in the infinite lattice may be expressed in terms of a lattice vector with origin $o$. Now, seeking for the pressure field at any point in the infinite array we can use the Bloch-Floquet periodic condition, which is given by

$$
p\left(\mathbf{r}+\mathbf{R}_{j}\right)=\exp \left(i \beta^{T} \mathbf{R}_{j}\right) p(\mathbf{r}),
$$

where $\boldsymbol{\beta}=\left(\beta_{x}, \beta_{y}\right)$ is the Bloch wave vector and $p(\mathbf{r})$, the pressure at field point $\mathbf{r}=(x, y)$. Periodicity implies that $p\left(\mathbf{r}+\mathbf{R}_{j}\right)$ can be expressed in terms of $p(\mathbf{r})$ when multiplied with a phase-factor. This allows one to obtain properties of an infinite array by utilizing the periodicity of the problem.

In this work, FEM has been selected to solve for band diagrams of an infinite arrangement of scattering objects. In brief, one unit cell needs to be modeled, with periodic boundary conditions applied to the edges of the computational domain. Two sets of periodic conditions are introduced, where each set is formed by two opposite domainedge sides. The crux of constructing band diagrams using FEM is to find (complex) eigenvalues of the system, when applied to certain Bloch wave-vectors. Corresponding eigenfrequencies can be written in the form $\omega=\omega_{0}+\delta$, where the second term is proportional to damping. Due to symmetry it is sufficient to restrict the variation of Bloch wave-vectors to the $\Gamma-X-M$ contour, i.e., the well-known reduced zone. The corner wave-number coordinates are $\Gamma=(0,0), X=\left(\pi / a_{1}\right.$, $0)$, and $M=\left(\pi / a_{1}, \pi / a_{2}\right)$. All band structure computations are performed with a total of $3 \times 50$ Bloch wave-vectors, i.e., 50 per propagation direction.

Here, the perforated shell for the infinite structures are modeled by an effective sound speed, ${ }^{9}$

$$
c_{l}=\frac{h}{h+\left(\delta_{i}+\delta_{o}\right)} c_{0},
$$

and effective density, ${ }^{9}$

$$
\rho_{l}=\frac{h+\left(\delta_{i}+\delta_{o}\right)}{h \phi} \rho_{0} .
$$

These effective propagation constants have originally been derived for slotted cylinders, though are applicable for perforated cylinders if the appropriate set of end-correction terms and perforation ratio are selected.

\section{B. Calculation results of finite and infinite arrays}

We will now present calculation results of finite and infinite arrays, and compare band diagrams as well as insertion loss spectra of acoustically rigid cylinders, perforated cylinders, and perforated cylinders with a porous core. Moreover, a number of internal and external mode shapes are shown to explain the phenomena giving rise to gaps in the transmission spectra.

Band diagrams and insertion loss spectra of perforated cylinders without a porous core and acoustically rigid cylinders are shown in Fig. 7(a). It can be seen that the insertion loss maxima for perforated cylinders (black curve), correspond well with the first three band-gaps highlighted for the $\Gamma-X$ direction. The first complete band-gap for perforated cylinders starts at around $821.3 \mathrm{~Hz}$, and is associated to the $(0,1)$ internal mode shown in Fig. 7(b).

The second band-gap is a partial gap and is related to an external mode as shown in Fig. 7(c). In addition, the $(1,1)$ internal mode is plotted in Fig. 7(d). The latter is associated with the second internal resonance at around $4636.6 \mathrm{~Hz}$,

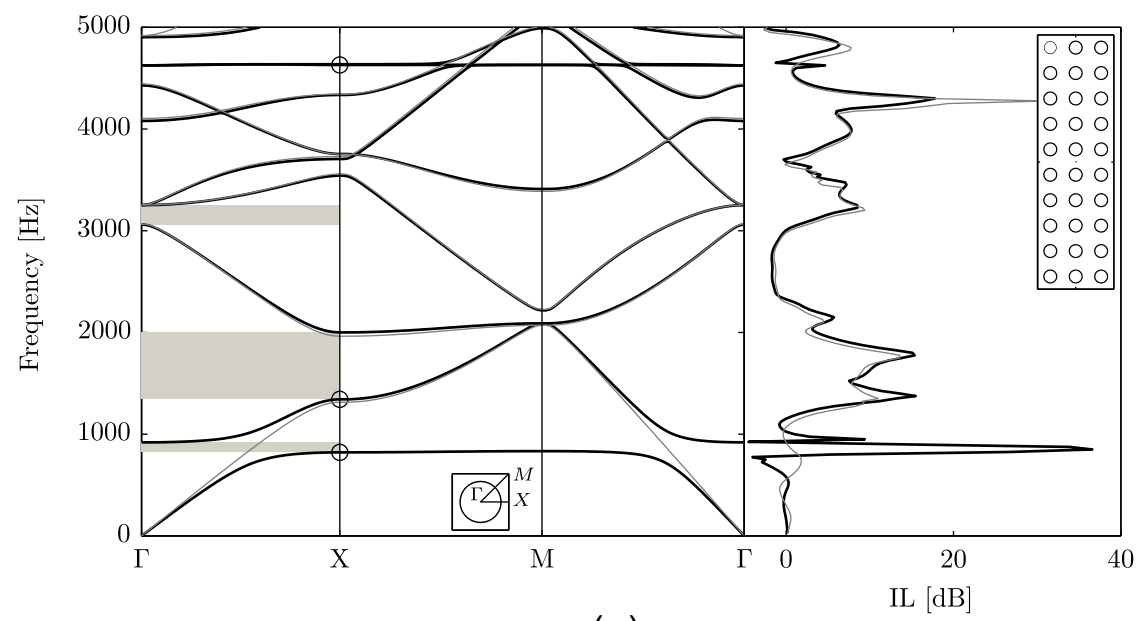

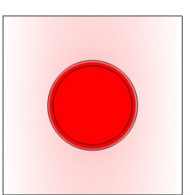

(b)

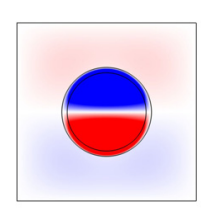

(d)

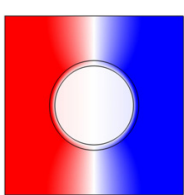

(c)

(a)

FIG. 7. (Color online) (a) Band diagram of infinite periodic array of perforated cylindrical shells and acoustically rigid cylinders (left panel), compared against the finite $10 \times 3$ arrays (right panel). Band diagrams of perforated cylinders (solid-black) and acoustically rigid cylinders (thin-gray) are plotted. The first three band-gaps in the $\Gamma$-X direction are marked in gray. (b)-(d) Mode shapes of an infinite array of perforated cylinders, where (b) is an internal mode at $821.3 \mathrm{~Hz}$, (c) an external mode at $1342.3 \mathrm{~Hz}$, (d) an internal mode at $4636.6 \mathrm{~Hz}$. 
which only spans over a narrow frequency interval. For acoustically rigid cylinders the gaps due to internal resonances are obviously non-existing, though otherwise the system behavior is comparable to the perforated shells.

We now compare band diagrams and insertion loss spectra of perforated cylinders with and without a porous core, see Fig. 8(a). Band diagrams of cylinders with a porous core are computed for $\sigma=15.1 \mathrm{kPa} \mathrm{s} \mathrm{m}^{-2}$, whereas insertion loss spectra are computed for $\sigma=9,15.1$, and $21 \mathrm{kPa} \mathrm{s} \mathrm{m}^{-2}$. It can be seen that adding porous material in the core of the scatterer will simultaneously damp and broaden the resonance peak. In addition, the resonance peak location is shifted down in frequency by approximately $75 \mathrm{~Hz}$. Increasing the flow resistance of the porous material will mainly suppress the damping peak as can be seen in the right panel of Fig. 8(a).

Comparing band diagrams of the two cylinder types reveal that the second resonance at around $4640 \mathrm{~Hz}$ is damped by adding porous material in the core of the scatterer. In addition, it can be seen that the band diagrams of both systems are approximately the same from and above the second band-gap. Band gaps associated with Bragg diffraction from the lattice thus remain to exist for perforated cylinders with porous cores. The first internal and external mode shapes for perforated cylinders with a porous core are found to be similar to the ones found for ordinary perforated cylinders, see Figs. 7(b), 7(c) and Figs. 8(b), 8(c). However, most of the eigenvalues of the former case are now found to be complex. The imaginary part of $\omega$ is generally small, though increases towards the fundamental resonance frequency with a maximum of $\omega / 2 \pi=734.2+36.8 i \mathrm{~Hz}$. The corresponding mode shape is shown in Fig. 8(b). As $\delta \ll \omega_{0}$, we can assume small damping and present $|\omega / 2 \pi|$ in the left panel of Fig. 8 .

\section{Reflectance, transmittance and absorption spectra as function of perforation ratio}

So far, the perforation ratio of the shell, the shell thickness, and the perforation radius has been kept constant. The insertion loss behind an array of perforated cylinders with a porous core, as studied in previous sections, could likely be improved by finding the optimum settings of these parameters. In addition, the cylinder arrangement and type of core material are parameters to consider while searching for an optimum configuration. The authors do, however, believe that there is no room for such an extensive search in the present work; hence we will not seek for a set of optimum parameters, but rather focus on the perforation ratio and its role onto the transmittance, reflectance, and absorption spectra. As such, we can distinguish the contribution of absorption by the porous core, and attenuation (behind the structure) due to reflection towards the source-side. Readers who need to find optimum parameters for specific (geometrical) configurations are referred to, e.g., Ref. 33, where a comprehensive optimization procedure using a multiobjective genetic algorithm is explained in detail. We will start with a geometrical setup using $150 \times 3$ cylindrical units, organized in square lattice, having a lattice constant $a=10 \mathrm{~cm}$. The array is oriented as in previous sections, and irradiated by a plane wave propagating perpendicular to sample surface. Properties of the cylindrical units are as before, with a porous core characterized by $\sigma=15.1 \mathrm{kPa} \mathrm{s} \mathrm{m}^{-2}$. The perforation ratio of the shells are set to $1 \%, 2 \%$, and $4 \%$, where the upper limit is chosen such that the perforation ratio remains small and holeinteraction effects can be neglected. The normal incidence reflectance spectra are here computed by

$$
\mathcal{R}=\left(\frac{\sum_{i=1}^{N_{r}}\left|p_{o, i}^{s}\right|}{\sum_{i=1}^{N_{r}}\left|p_{o, i}^{d}\right|}\right)^{2}
$$

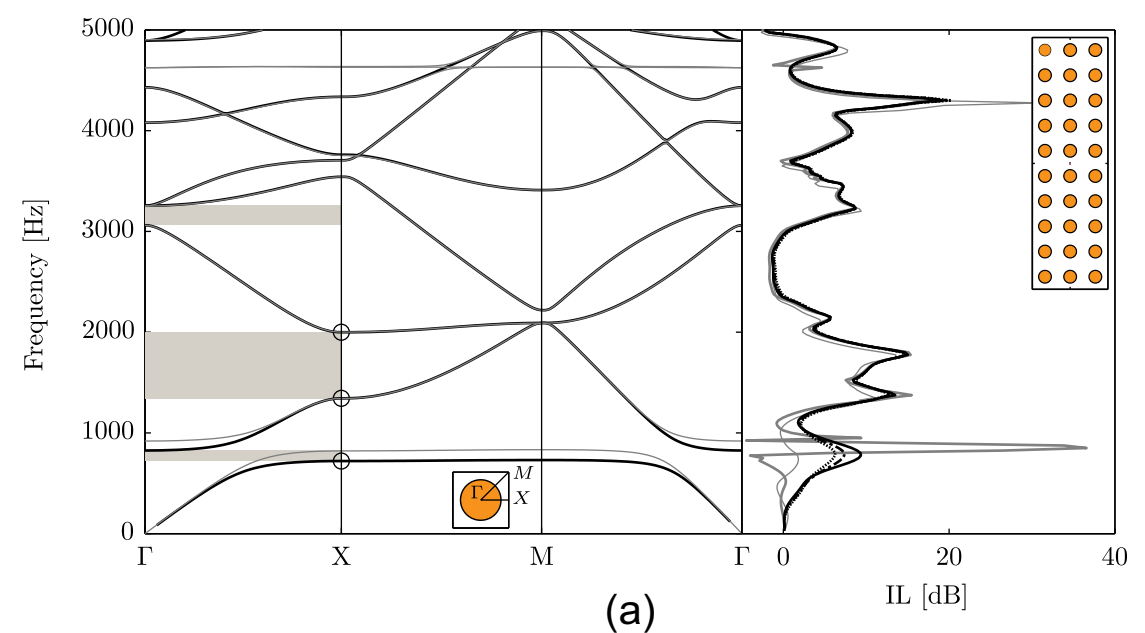

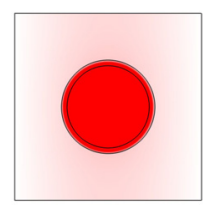

(b)

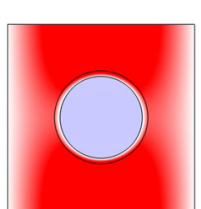

(d)

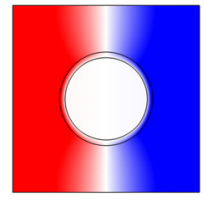

(c)

FIG. 8. (Color online) (a) Band diagram of infinite periodic array of perforated cylindrical shells with porous core using $\sigma=15.1 \mathrm{kPa} \mathrm{s} \mathrm{m}^{-2}$, and without a porous core (left panel), compared against the finite $10 \times 3$ arrays (right panel). Band diagrams of perforated cylinders with porous core (black) and perforated cylinders without porous core (gray) are plotted. The first three band-gaps in the $\Gamma$ - $X$ direction are marked in gray. In the right panel of (a) we can distinguish: hard cylinders (thin-gray), perforated cylinders (thick-gray), and perforated cylinders with a porous core using $\sigma=9,15.1$, and $21 \mathrm{kPa} \mathrm{s} \mathrm{m}^{-2}$, which are depicted in solid-black, dashed-black, and dotted-black, respectively. (b)-(d) Mode shapes of an infinite array of perforated cylinders with porous core, where (b) is an internal mode at $734.2+36.8 \mathrm{i} \mathrm{Hz}$, (c) an external mode at $1344.0-0.4 \mathrm{i} \mathrm{Hz}$, (d) and an external mode at $1999.3+0.8 \mathrm{i} \mathrm{Hz}$. 


$$
\mathcal{T}=\left(\frac{\sum_{i=1}^{N_{r}}\left|p_{o, i}\right|}{\sum_{i=1}^{N_{r}}\left|p_{o, i}^{d}\right|}\right)^{2},
$$

where, $p_{o, i}^{s}$ is the scattered field, $p_{o, i}^{d}$ the incoming field and $p_{o, i}$ the total field of the $i$ th receiver. To compute $\mathcal{R}$ and $\mathcal{T}$, we perform a summation over a number of receivers $N_{r}$. The first receivers are placed just outside the array, i.e., on the source-side at $\left(x_{r}, z_{r}\right)=(-0.16,0) \mathrm{m}$ and the receiver-side at $(0.16,0) \mathrm{m}$, where the origin of the array is now placed at $(x, z)=(0,0) \mathrm{m}$. On both sides of the array a line of 50 receivers is placed along the $x$ axis, with a receiver separation of $1 \mathrm{~cm}$. The normal incidence sound absorption spectra are than computed through conservation of energy, i.e., $\mathcal{A}=1-\mathcal{R}-\mathcal{T}$. However, this approach is only valid below the diffraction limit, i.e., the frequency interval marking the existence of acoustic scattering solely in the direction of excitation, see, e.g., Ref. 16. The diffraction limit is theoretically found when $\lambda=a$, here at around $3.4 \mathrm{kHz}$, though band-structure calculations suggest a slightly lower frequency of about $3.25 \mathrm{kHz}$. We therefore restrict the analysis to an upper frequency of $3 \mathrm{kHz}$. Absorption, transmittance, and reflectance spectra are shown in Fig. 9.

In Fig. 9, it can be seen that by increasing the perforation ratio of the shell a globally substantial increase of energy dissipation may be obtained. However, this holds true only if the perforation ratio and the shell thickness are chosen such that absorption peak is well within the frequency range of interest. That is, e.g., increasing the perforation ratio of the shell will simultaneously increase the resistive and reactive end-correction terms; the latter will cause a shift of the fundamental resonance up to a higher frequency. This phenomenon is also captured in the plotted absorption spectra, where we observe a frequency shift of the peak absorption by increasing the perforation ratio of the shell. Due to an increase in energy dissipation the total
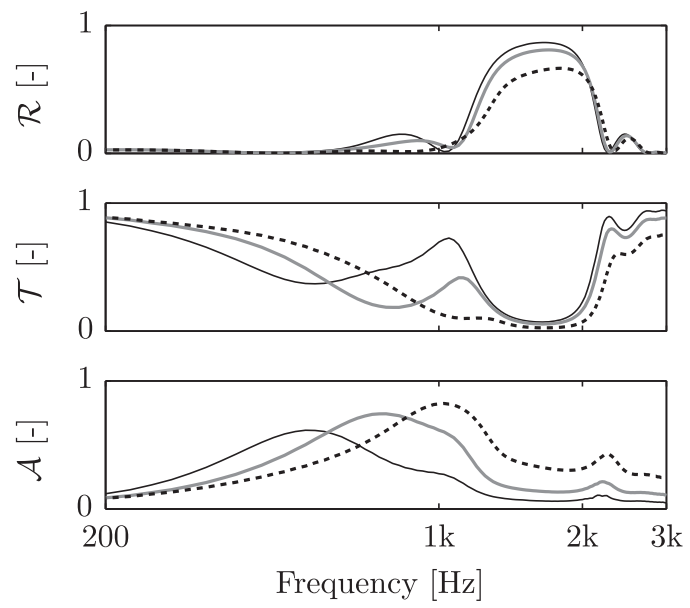

FIG. 9. Absorption, reflectance and transmittance spectra for an array of $150 \times 3$ perforated units with a porous core, organized in square lattice, having a lattice constant $a=10 \mathrm{~cm}$. The porous core is characterized by $\sigma=15.1 \mathrm{kPa} \mathrm{s} \mathrm{m}^{-2}$. We distinguish: a shell-perforation ratio of $1 \%$ (thinblack), $2 \%$ (solid-gray), and $4 \%$ (dashed). energy transport through the array is mostly reduced, as can be concluded by comparing the transmittance spectra as shown. It is also worth mentioning that, by increasing the perforation ratio, backscattering towards the source-side is substantially reduced around the band-gap frequency interval, while energy transport through the sample is lowered.

\section{DISCUSSION}

Multiple scattering by an array of perforated cylinders with and without a porous core has been presented in this paper. In order to predict scattering from such configurations, a semi-analytical model developed in the context of MST was introduced and verified with laboratory experiments. The agreement between measured and predicted insertion losses of a small array was found to be very good. By mounting the array onto a computer controlled turntable several rotational orientations could be measured, and compared against calculations results. The agreement for all array orientations was found to be of similar quality. Having the ability to rotate the array in discrete steps, we found that vertical alignment of the cylinders is crucial for good results. That is, a slightly skewed alignment of the array may lead to significant shifts in the insertion loss spectra, particularly at higher frequencies. Furthermore, it may be observed that the presented measurements are somewhat noisy in certain frequency intervals, e.g., in Fig. 6(a) between 3-4 kHz. The applied transfer function measurement technique ensures, however, that these spectral oscillations are higher-order effects, e.g., due to interaction of perforated cylinders with surrounding objects. Additional damping material around the supporting structure did not improve nor worsen the spectral oscillations.

The band structure diagrams as presented are constructed by searching for (complex) eigenvalues of a FEM meshed square unit cell, with two pairs of Bloch-Floquet period boundary conditions applied to the domain edges. The method allows one to search for damped propagating modes in a straightforward manner as the imaginary part of $\omega$ is linked to damping of the system-a system here being an infinite array of two-dimensional resonators with or without a porous core. We limited the band-structure evaluation to real Bloch wavevectors only. However, extending the domain to complex Bloch wave-vectors, one could, e.g., visualize the evanescent behavior at frequency intervals marking a band gap. ${ }^{34,35}$

For the sake of simplicity we have constructed cylinders with circular perforations and used glass wool to make porous cores. However, other core materials as well as shell perforations could be modeled if suitable impedance expressions are at hand, see, e.g., Ref. 16. This in particular is needed for constructing, e.g., noise barriers, as glass-wool itself might be impractical for outdoor use. Note also that the perforation ratio and choice of porous material have not been optimized to maximize the insertion loss, though adjusting the perforation ratio of the shell did suggest there is room for improvement.

\section{CONCLUSIONS}

In this paper it is shown that adding porous material in the core of a perforated cylinder will simultaneously broaden 
and lower the fundamental resonance peak of the resonator. It is also found that after adding porous material inside the perforated shell the interior resonance is shifted down in frequency.

Substantial noise reduction is obtained in wide frequency range around the main resonance peak, which was targeted to be in the low-frequency region. In this frequency region it is otherwise difficult to mitigate noise by using period arrangements of scatterers. Above the fundamental resonance frequency, the insertion loss spectra as well as band diagrams of the infinite systems were practically indistinguishable with those of the acoustically rigid scatterers. It has, however, been shown that energy transport through the (finite) array is partially controlled by energy dissipation of the porous core, which could be further improved by adjusting the perforation ratio of the shells.

\section{ACKNOWLEDGMENTS}

The research leading to these results has received funding from the European Community's Seventh Framework Programme (FP7/2007-2013) under Grant agreement No. 234306, collaborative project HOSANNA.

${ }^{1}$ F. Cervera, L. Sanchis, J. Sánchez-Pérez, R. Martínez-Sala, C. Rubio, F. Meseguer, C. López, D. Caballero, and J. Sánchez-Dehesa, "Refractive acoustic devices for airborne sound," Phys. Rev. Lett. 88(2), 023902 (2001).

${ }^{2}$ Bart van der Aa and Jens Forssén, "Upward refraction of sound propagating outdoors by a graded index sonic crystal noise barrier," Appl. Acoust. 74(1), 89-101 (2013).

${ }^{3}$ D. Caballero, J. Sanchez-Dehesa, C. Rubio, R. Martinez-Sala, J. V. Sanchez-Perez, F. Meseguer, and J. Llinares, "Large two-dimensional sonic band gaps,” Phys. Rev. E 60(6), R6316 (1999).

${ }^{4}$ You-Yu Chen and Zhen Ye, "Acoustic attenuation by two-dimensional arrays of rigid cylinders," Phys. Rev. Lett. 87, 184301 (2001).

${ }^{5}$ J. V. Sánchez-Pérez, D. Caballero, R. Martinez-Sala, C. Rubio, J. Sánchez-Dehesa, F. Meseguer, J. Llinares, and F. Gálvez, "Sound attenuation by a two-dimensional array of rigid cylinders," Phys. Rev. Lett. 80(24), 5325-5328 (1998).

${ }^{6}$ R. Martinez-Sala, J. Sancho, J. V. Sanchez, V. Gomez, J. Llinares, and F. Meseguer, "Sound attenuation by sculpture," Nature (London) 378, 241 (1995).

${ }^{7}$ Daniel, P. Elford, Luke Chalmers, Feodor V. Kusmartsev, and Gerry M. Swallow, "Matryoshka locally resonant sonic crystal," J. Acoust. Soc. Am. 130(5), 2746-2755 (2013).

${ }^{8}$ Xinhua Hu, C. Chan, and Jian Zi, "Two-dimensional sonic crystals with Helmholtz resonators," Phys. Rev. E 71(5), 055601 (2005).

${ }^{9}$ Anton Krynkin, Olga Umnova, Alvin Y. B. Chong, Shahram Taherzadeh, and Keith Attenborough, "Scattering by coupled resonating elements in air," J. Phys. D: Appl. Phys. 44(12), 125501 (2011).

${ }^{10} \mathrm{C}$. Lagarrigue, J. P. Groby, and V. Tournat, "Sustainable sonic crystal made of resonating bamboo rods," J. Acoust. Soc. Am. 133, 247-254 (2013).

${ }^{11}$ V. Romero-García, A. Krynkin, L. M. Garcia-Raffi, O. Umnova, and J. V. Sánchez-Pérez, "Multi-resonant scatterers in sonic crystals: Locally multiresonant acoustic metamaterial," J. Sound Vib. 332(1), 184-198 (2013).

${ }^{12}$ Anton Krynkin and Olga Umnova, "Predictions and measurements of sound transmission through a periodic array of elastic shells in air," J. Acoust. Soc. Am. 128, 3496-3506 (2010).
${ }^{13}$ Victor M. García-Chocano, Suitberto Cabrera, and José Sánchez-Dehesa, "Broadband sound absorption by lattices of microperforated cylindrical shells," Appl. Phys. Lett. 101(18), 184101 (2012).

${ }^{14}$ Olga Umnova, Keith Attenborough, and Chris M. Linton, "Effects of porous covering on sound attenuation by periodic arrays of cylinders," J. Acoust. Soc. Am. 119(1), 278-284 (2006).

${ }^{15}$ V. Romero-García, J. V. Sánchez-Pérez, and L. M. Garcia-Raffi, "Tunable wideband band-stop acoustic filter based on two-dimensional multiphysical phenomena periodic systems," J. Appl. Phys. 110(1), 014904 (2011).

${ }^{16}$ José Sánchez-Dehesa, Victor M. Garcia-Chocano, Daniel Torrent, Francisco Cervera, Suitberto Cabrera, and Francisco Simon, "Noise control by sonic crystal barriers made of recycled materials," J. Acoust. Soc. Am. 129, 1173-1183 (2011).

${ }^{17}$ Xinhua Hu, Kai-Ming Ho, C. T. Chan, and Jian Zi, "Homogenization of acoustic meta-materials of Helmholtz resonators in fluid," Phys. Rev. B 77, 172301 (2008).

${ }^{18}$ Feiyan Cai, Fengming Liu, Zhaojian He, and Zhengyou Liu, "High refractive-index sonic material based on periodic subwavelength structure," Appl. Phys. Lett. 91, 203515 (2007).

${ }^{19}$ Jean F. Allard, Propagation of Sound in Porous Media: Modelling Sound Absorbing Materials (Elsevier Applied Science, London, 1993), pp. 220-253.

${ }^{20}$ Dah-You Maa, "Potential of microperforated panel absorber," J. Acoust. Soc. Am. 104(5), 2861-2866 (1998).

${ }^{21}$ Rolf Tore Randeberg, "Perforated panel absorbers with viscous energy dissipation enhanced by orifice design," Ph.D. thesis, Norwegian University of Science and Technology, 2000.

${ }^{22}$ Uno Ingard, "On the theory and design of acoustic resonators," J. Acoust. Soc. Am. 25(6), 1037-1061 (1953).

${ }^{23}$ Noureddine Atalla and Franck Sgard, "Modeling of perforated plates and screens using rigid frame porous models," J. Sound Vib. 303(1-2), 195-208 (2007).

${ }^{24}$ Uno Ingard and R. H. Bolt, "Absorption characteristics of acoustic material with perforated facings," J. Acoust. Soc. Am. 23(5), 533-540 (1951).

${ }^{25} \mathrm{R}$. Kirby and A. Cummings, "The impedance of perforated plates subjected to grazing gas flow and backed by porous media," J. Sound Vib. 217(4), 619-636 (1998).

${ }^{26}$ M. E. Delany and E. N. Bazley, "Acoustical properties of fibrous absorbent materials," Appl. Acoust. 3(2), 105-116 (1970).

${ }^{27}$ Yasushi Miki, "Acoustical properties of porous materials-modifications of Delany-Bazley models," J. Acoust. Soc. Jpn. 11(1), 19-28 (1990).

${ }^{28} \mathrm{D}$. Keith Wilson, "Simple, relaxational models for the acoustical properties of porous media," Appl. Acoust. 50(3), 171-188 (1997).

${ }^{29}$ Bikash Gupta and Zhen Ye, "Theoretical analysis of the focusing of acoustic waves by two-dimensional sonic crystals," Phys. Rev. E 67(3), 036603 (2003).

${ }^{30}$ L. Sanchis, F. Cervera, J. Sánchez-Dehesa, J. V. Sánchez-Pérez, C. Rubio, and R. Martínez-Sala, "Reflectance properties of two-dimensional sonic band-gap crystals," J. Acoust. Soc. Am. 109(6), 2598-2605 (2001).

${ }^{31}$ J. Y. Chung and D. A. Blaser, "Transfer function method of measuring in-duct acoustic properties. I. Theory,” J. Acoust. Soc. Am. 68, 907-913 (1980).

${ }^{32}$ Ray Kirby, "On the modification of Delany and Bazley formulae," Appl. Acoust. 86, 47-49 (2014)

${ }^{33}$ Bart van der Aa and Jens Forssén, "Shape-optimal design of graded index sonic crystal formations using natural cubic splines," Appl. Acoust. 78, 98-111 (2014).

${ }^{34}$ Christian Engström, Christian Hafner, and Kersten Schmidt, "Computations of lossy Bloch waves in two-dimensional photonic crystals," J. Comput. Theor. Nanosci. 6(3), 775-783 (2009).

${ }^{35}$ V. Romero-García, L. M. Garcia-Raffi, and J. V. Sánchez-Pérez, "Evanescent waves and deaf bands in sonic crystals," AIP Adv. 1(4), 041601 (2011). 